\title{
Detection of the binding the stress HliA protein Synechocystis sp. with pigments
}

\author{
Sharapova L.S.*, Yurina N.P. \\ Bach Institute of Biochemistry, Federal Research Center "Fundamentals of Biotechnology" of the Russian \\ Academy of Sciences, Moscow, Russia \\ * email: sharapova0902@mail.ru
}

The high-light-inducible (Hli) proteins of cyanobacteria is considered as the evolutionary precursors of chlorophyll a/b-binding proteins of plants and algae. It has been shown that Hlips are essential for cell survival under intense light conditions, are involved in the regulation of chlorophyll biosynthesis, transport and binding of free chlorophyll molecules, quenching of singlet oxygen, assembly and repair of photosystem II, and non-photochemical dissipation of absorbed light energy. However, the full picture of the functioning and localization of Hli proteins is not fully understood. To protect cells from excessive light, an important process is the binding of phototoxic free chlorophyll molecules that cause photodestruction and oxidative stress. Hli proteins can serve as such key binding agents for these molecules. Four Hli proteins (HliA, B, C, and D) were found in cyanobacteria Synechocystis sp. PCC 6803. Previously, these proteins were isolated as part of small complexes with other photosystem proteins. In this paper, for the first time, the isolation and characterization of the pure HliA protein Synechocystis sp. A recombinant plasmid containing the HliA gene with $6 \mathrm{His}$ at the Cterminus (HliA-6His) of the protein was obtained. The pure recombinant HliA protein of Synechocystis sp was isolated by Ni-chelate chromatography. The HliA protein was reconstituted with chlorophyll a and carotenoids. Using circular dichroism spectroscopy, it was shown that chlorophyll a and carotenoids interact in vitro with the HliA protein. The binding of pigments to the HliA protein indicates the protective role of this protein. The HliA protein was found to be important for the oxygen uptake reaction in photosystem I with an artificial donor and acceptor. The necessity of HliA for optimal photochemical activity of the photosystem I complex is shown. The results are important for understanding photoprotection processes in both cyanobacteria and algae and higher plants.

Acknowledgements: This work was partially supported by the Russian Foundation for Basic Research (Grant No. 19-04-00798). 